\title{
H I in Group Interactions: HCG 44
}

\author{
Kelley M. Hess ${ }^{1,2},{ }^{\star}$ M. E. Cluver ${ }^{3}$, Sahba Yahya ${ }^{3}$, Lukas Leisman ${ }^{4}$, Paolo Serra ${ }^{5}$, \\ Danielle M. Lucero ${ }^{1}$, Sean S. Passmoor ${ }^{6}$, and Claude Carignan ${ }^{7,8}$ \\ ${ }^{1}$ Kapteyn Astronomical Institute, University of Groningen, Landleven 12, 9747 AD, Groningen, The Netherlands \\ ${ }^{2}$ ASTRON, the Netherlands Institute for Radio Astronomy, Postbus 2, 7990 AA, Dwingeloo, The Netherlands \\ ${ }^{3}$ Physics Department, University of the Western Cape, Cape Town 7535, South Africa \\ ${ }^{4}$ Cornell Center for Astrophysics and Planetary Science, Space Sciences Building, Cornell University, Ithaca, NY 14853, USA \\ ${ }^{5}$ CSIRO Astronomy and Space Science, Australia Telescope National Facility, PO Box 76, Epping, NSW 1710, Australia \\ ${ }^{6}$ Square Kilometre Array South Africa, The Park, Park Road, Pinelands 7405, South Africa \\ ${ }^{7}$ Department of Astronomy, University of Cape Town, Private Bag X3, Rondebosch 7701, South Africa \\ ${ }^{8}$ Laboratoire de Physique et Chimie de l'Environnement (LPCE), Observatoire d'Astrophysique de l'Université de Ouagadougou, BP 7021, \\ Ouagadougou 03, Burkina Faso
}

Accepted 2016 September 13. Received 2016 September 12; in original form 2016 May 26

\begin{abstract}
Extending deep observations of the neutral atomic hydrogen (H I) to the environment around galaxy groups can reveal a complex history of group interactions which is invisible to studies that focus on the stellar component. Hickson Compact Group 44 (HCG 44) is a nearby example and we have combined H I data from the Karoo Array Telescope, Westerbork Synthesis Radio Telescope, and Arecibo Legacy Fast ALFA survey, in order to achieve high column density sensitivity $\left(N_{H I}<2 \times 10^{18} \mathrm{~cm}^{-2}\right)$ to the neutral gas over a large field-of-view beyond the compact group itself. We find the giant $\mathrm{H}$ I tail north of HCG 44 contains $1.1 \times 10^{9} \mathrm{M}_{\odot}$ of gas and extends $450 \mathrm{kpc}$ from the compact group: twice as much mass and $33 \%$ further than previously detected. However, the additional gas is still unable to account for the known H I deficiency of HCG 44. The tail likely formed through a strong tidal interaction and H I clouds in the tail have survived for $1 \mathrm{Gyr}$ or more after being stripped. This has important implications for understanding the survival of neutral clouds in the intragroup and circumgroup medium, and we discuss their survival in the context of simulations of cold gas in hot halos. HCG 44 is one of a growing number of galaxy groups found to have more extended $\mathrm{HI}$ in the intragroup and circumgroup medium than previously measured. Our results provide constraints for simulations on the properties of galaxy group halos, and reveal a glimpse of what will be seen by future powerful $\mathrm{H}$ I telescopes and surveys.
\end{abstract}

Key words: galaxies: evolution - galaxies: interactions - galaxies: ISM - radio lines: galaxies - galaxies: groups: individual: HCG 44

\section{INTRODUCTION}

Large area redshift surveys such as SDSS (Abazajian et al. 2009) and GAMA (Driver et al. 2009) have established a new paradigm regarding the clustering of galaxies (Berlind et al. 2006; Robotham et al. 2011). The evolutionary effects imposed by this range of environments are not fully understood, largely due to the complexity of N-body interactions on star formation and gas dynamics. However, the relatively unexplored regime of neutral $\mathrm{HI}$ in the context of galaxy groups has the potential to revolutionise our

* E-mail: hess@astro.rug.nl understanding of the mechanisms influencing how galaxies mature.

Targeted observations of the Hi content in nearby groups have often revealed the presence of intragroup material, for example, with the Very Large Array (VLA e.g. van Moorsel 1988; Yun et al. 1994), the Parkes radio telescope (e.g. Kilborn et al. 2006), the Australia Telescope Compact Array (e.g. Barnes \& Webster 2001; Koribalski \& Dickey 2004), and Nançay (van Driel et al. 2001). However, systematic surveys of galaxy groups are rare due to the observational time necessary to achieve the required sensitivities, even at relatively modest redshifts, over large fields of view. The blind, wide-area Arecibo Legacy Fast ALFA (ALFALFA) survey (Giovanelli et al. 
2005; Haynes et al. 2011; Huang et al. 2012), combined with the Sloan Digital Sky Survey (SDSS) DR7 group catalogue (Berlind et al. 2006; Berlind \& SDSS 2009), enabled Hess \& Wilcots (2013) to explore the H I content of group galaxies as a function of optical group membership. They found evidence for gas pre-processing correlated with group assembly. Meanwhile, the Blind Ultra Deep HI Survey (BUDHIES) on the Westerbork Synthesis Radio Telescope (WSRT), a targeted survey at $z \approx 0.2$, combines accurate $\mathrm{H}$ I content with morphology in environments ranging from voids to cluster centres to show evidence for both ram pressure and pre-processing as mechanisms for gas removal (Jaffé et al. 2013, 2015).

The role of compact groups, in particular, has long been advocated for understanding galaxy evolution processes: their high galaxy densities combined with relatively shallow potential wells prolongs strong gravitational interactions. Hickson (1982) defined compact groups as having 4 or more members within a 3 magnitude range $(\delta \mathrm{mB})$ that form a physically isolated system. The original Hickson catalogue has been modified based on radial velocity information with 92 groups having at least three accordant members (Hickson et al. 1992). Although other compact group catalogues exist, it remains the most intensively studied sample.

The H I deficiency in compact group galaxies (Williams \& Rood 1987; Huchtmeier 1997) was the focus of a single-dish study of 72 HCGs by Verdes-Montenegro et al. (2001). In combination with a subset resolved by the VLA, the authors proposed an evolutionary sequence where galaxies become increasingly $\mathrm{H}$ I deficient due to multiple tidal interactions. The study of compact group evolution was expanded by Green Bank Telescope (GBT) observations from Borthakur et al. (2010) which suggested the presence of copious diffuse, low column-density neutral hydrogen gas between the galaxies. However, even this emission is not always able to account for the HI deficiency of compact groups. Further studies suggest that the most evolved groups, which are also dominated by elliptical galaxies, begin to exhibit signs of hot intragroup gas due to gravitational heating (Ponman et al. 1996; Osmond \& Ponman 2004). However, strong H I deficiency does not guarantee the presence of intragroup X-ray emitting gas, complicating the interpretation of what mechanisms are responsible for removing the cold gas (Rasmussen et al. 2008; Desjardins et al. 2012).

Additional evidence of evolution within compact groups has come from photometry from the Spitzer Space Telescope: bimodality in IRAC color-color space, appearing to correlate with $\mathrm{HI}$ depletion, suggests accelerated evolution from dusty, star-forming galaxies, to gas-poor systems (Johnson et al. 2007; Walker et al. 2010). Detailed studies by Verdes-Montenegro et al. (1998); Tzanavaris et al. (2010); Walker et al. (2012) further support a picture of accelerated evolution. Evidence of shock-excited $\mathrm{H}_{2}$ in several compact group galaxies led Cluver et al. (2013) to suggest a connection between intragroup tidal debris and rapid quenching in these groups.

HCG 44 is a relatively nearby compact group with redshift $z \sim 0.0046$ and an angular diameter of approximately $16^{\prime}$. It contains four massive galaxies: three starforming spirals (NGC 3185, NGC 3187, NGC 3190), and one elliptical which is undetected in H I (NGC 3193); and the H I cloud, $C_{\mathrm{S}}$, which lacks an apparent optical counterpart (Serra et al. 2013). The galaxies are highly H Ideficient (Verdes-Montenegro et al. 2001), but X-ray emission is confined to individual galaxies with no detectable diffuse X-ray emission associated with the intragroup medium (Rasmussen et al. 2008), leaving the missing H I unaccounted for. Further, the lack of diffuse hot gas brings into question the importance of ram pressure stripping as a mechanism for removing gas from the large galaxies in this system. Cluver et al. (2013) found excited $\mathrm{H}_{2}$ in NGC 3190, perhaps shocked from impact with the intragroup medium, but GBT observations are unable to resolve individual galaxies, and high resolution interferometry observations have not yet detected intragroup $\mathrm{H}$ I within the core of the compact group. In fact, the evolutionary stage of the compact group has been debated as the H I content suggests it is more advanced, while the lack of intragroup optical light from stars, and intragroup X-rays suggest it is younger (Aguerri et al. 2006; Osmond \& Ponman 2004).

As part of the ATLAS ${ }^{3 \mathrm{D}}$ survey, Serra et al. (2012) surveyed the neutral gas content of HCG 44 and found evidence for an extended $\mathrm{H}$ I distribution well beyond the compact group. Deeper WSRT data, combined with reprocessed HIPASS data, detected the presence of a $\sim 300 \mathrm{kpc}$ long tail to the north of HCG 44 containing $5 \times 10^{8} M_{\odot}$ of $\mathrm{H} \mathrm{I}$ (Serra et al. 2013). The authors concluded that this tail is most likely the result of tidal interactions and proposed possible scenarios. First, the tail is the result of a high speed interaction between NGC 3162 and the compact group which occurred up to 3 Gyr in the past. NGC 3162 lies $~ 1.4$ deg (620 kpc at the assumed distance) northwest of HCG 44. Second, the tail is a result of tidal stripping due to NGC 3187 interacting with the gravitational potential of the compact group, which would explain its very disturbed optical morphology. However, they noted that deeper H I data over a larger field would benefit detailed modeling of this system.

In this paper we investigate the extended region around HCG 44 and the northern tail by conducting a deep H I mosaic with the Karoo Array Telescope (KAT-7) which, due to its sensitivity and resolution, is ideal for detecting low column density H I gas (Carignan et al. 2013). We combine these data with the deep WSRT pointing from Serra et al. (2013), and an H I map spanning the same sky coverage from the ALFALFA survey to improve our overall picture of the $\mathrm{H}$ I distribution in the compact group, and nearby surroundings.

The paper is organized as follows. In Section 2 we describe the KAT-7 observations, and summarize the additional data. In Section 3 we describe our technique for combining interferometric images from KAT-7 and WSRT, and the single dish map from ALFALFA. In Section 4 we compare the measurements from KAT-7 and ALFALFA and present our results from the combined data cube. In Section 5 we discuss the possible origins and the fate of the extended H I tail. We conclude in Section 6.

\section{DATA}

\subsection{KAT-7 observations, and data reduction}

HCG 44 and the Hi tail ( $T_{\mathrm{N}}$; Serra et al. 2013) were observed with the KAT-7 telescope (Foley et al. 2016) in 


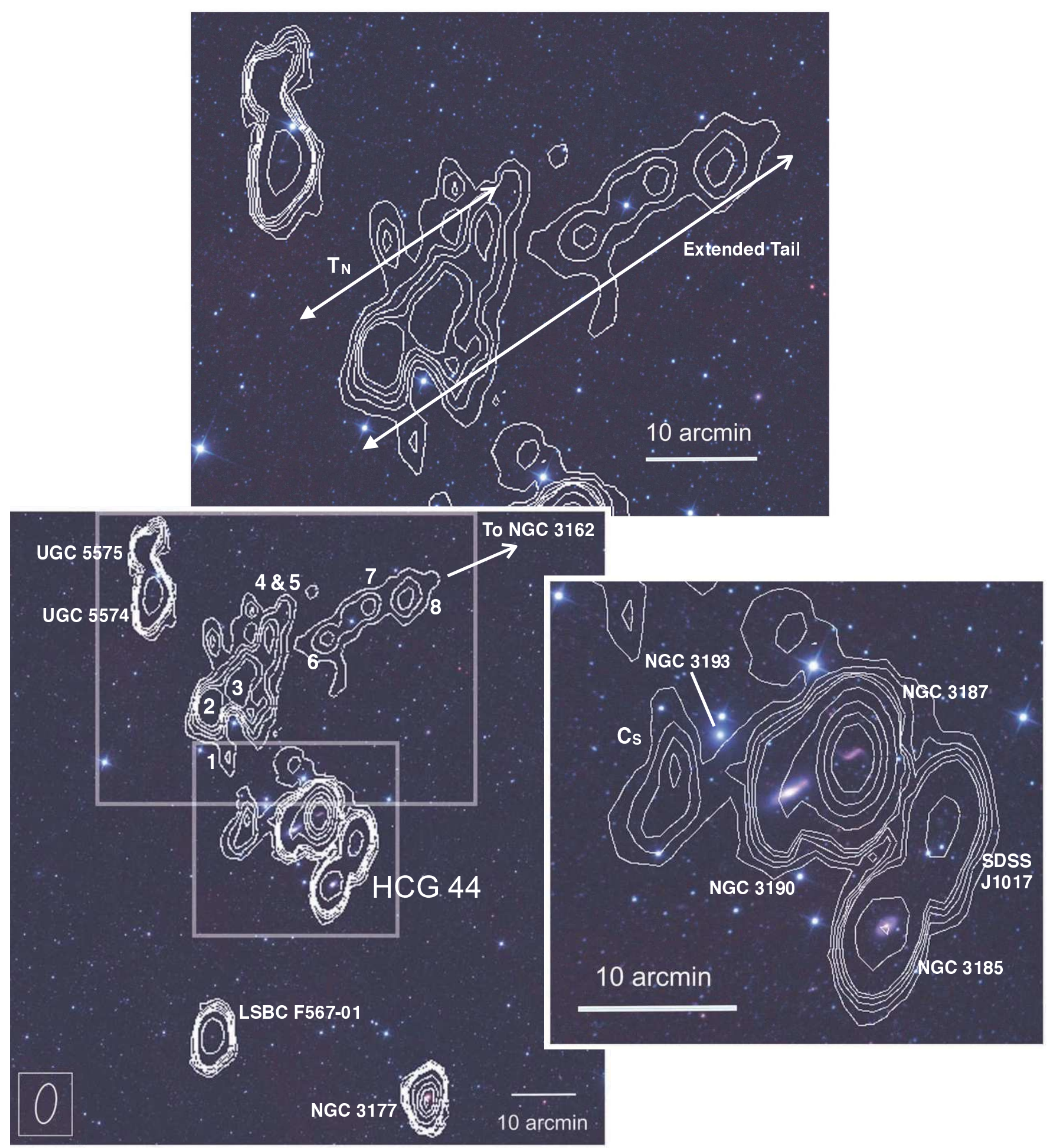

Figure 1. A "drizzled" Wide-field Infrared Survey Explorer (WISE) four-color image of HCG 44 and the extended H I tail: $3.4 \mu \mathrm{m}$ (W1) is blue, $4.6 \mu \mathrm{m}$ (W2) is green, $12 \mu \mathrm{m}$ (W3) is orange, and $22 \mu \mathrm{m}$ (W4) is red. White contours correspond to H I column densities of 2,4 , $6,8,10,25,50,75,100 \times 10^{18} \mathrm{~cm}^{-2}$ from the KAT-7 observations, and we have labeled the major H I features. H I clouds 4 and 5 in $T_{\mathrm{N}}$ are not well resolved in KAT-7, but are resolved in WSRT data (see Figure 2). Star forming galaxies appear red. Foreground Milky Way stars and old stellar populations appear blue. There is no evidence for a diffuse stellar counterpart to the H I in WISE imaging outside of the known galaxies. 
two pointings: one centered on the group itself ( $\alpha=$ $10^{\mathrm{h}} 17^{\mathrm{m}} 46.03^{\mathrm{s}}+21^{\circ} 48^{\prime} 30.8^{\prime \prime}$; hereafter Field 1 ), and one centered on the northern tail discovered by Serra et al. (2013) $\left(\alpha=10^{\mathrm{h}} 17^{\mathrm{m}} 54.312^{\mathrm{s}}+22^{\circ} 20^{\prime} 37.1^{\prime \prime}\right.$; hereafter Field 2$)$. Field 1 was observed with all seven antennas in 11 sessions between 2015 Jan 9 - 2015 May 20 for a total of 30 hours on source. Field 2 was later observed with six available antennas in 8 sessions between 2015 July 3 - 2015 July 13 for a total of 36.25 hours on source. Field 1 was observed during the night, whereas Field 2 had primarily day time observations and was affected by solar interference which resulted in more intensive flagging during data reduction.

The KAT-7 data were reduced in CASA 4.4.0 (McMullin et al. 2007) using standard procedures. As a first step, we flagged all $u=0$ data to remove low level radio frequency interference (RFI) that is correlated when the fringe rate is equal to zero (see Hess et al. 2015 for a complete explanation). After flux and gain calibration, we performed continuum subtraction using a first order fit in the task UVCONTSUB and imaged each observation individually for quality assurance. Field 1 and Field 2 achieved an rms noise of 2.7 and $2.9 \mathrm{mJy}$ beam $^{-1}$, respectively, over $7.7 \mathrm{~km} \mathrm{~s}^{-1}$ channels, despite the differing total observing times between the fields. We performed self-calibration on the continuum data of the target field and applied it to the spectral line data. This generally produced a factor of 1.2 improvement in the rms noise, and up to a factor of 1.5 in individual data sets. The final mosaic improved to $1.7 \mathrm{mJy}^{\text {beam }}{ }^{-1}$, with a $358 \times 187$ arcsec beam, thanks to self calibration and overlap between the fields.

We converted this to column density using Equation 1 from Hibbard et al. 2001:

$N_{H I}=\frac{1.104 \times 10^{21} \mathrm{~cm}^{-2}}{\theta_{a} \times \theta_{b}} \frac{\int S \Delta v}{\mathrm{mJy} \mathrm{beam}^{-1} \mathrm{~km} \mathrm{~s}^{-1}}$

where $S$ is in mJy, $\theta_{a}$ and $\theta_{b}$ are the major and minor axes of the beam in arcseconds, and $\Delta v$ is the channel width in $\mathrm{km} \mathrm{s}^{-1}$. This corresponds to a $1 \sigma \mathrm{H} \mathrm{I}$ column density of $2.2 \times 10^{17} \mathrm{~cm}^{-2}$ channel $^{-1}$. Figure 1 shows the H I contours of the KAT-7 mosaic overlaid on a four-color image from the Wide-field Infrared Survey Explorer (WISE). We describe the method for generating these contours in Section 4 .

\subsection{WSRT data}

Serra et al. (2013) observed HCG 44 with the WSRT for $6 \times 12$ hours at a single pointing centered between the group and the H I tail $\left(\alpha=10^{\mathrm{h}} 18^{\mathrm{m}} 45.19^{\mathrm{s}}+21^{\circ} 59^{\prime} 30.5^{\prime \prime}\right.$. The observations achieved an rms of $0.23 \mathrm{mJy}^{\mathrm{beam}}{ }^{-1}$ with a beam of $53.0 \times 32.7$ arcsec over $8.2 \mathrm{~km} \mathrm{~s}^{-1}$ channels.

\subsection{ALFALFA data}

HCG 44 also falls in the portion of sky observed by the ALFALFA survey. A full description of the survey strategy can be found in Giovanelli et al. (2005) and Haynes et al. (2011) but, in short, ALFALFA is a two-pass, fixed-azimuth, drift scan survey covering the velocity range from -300 to $+18,000 \mathrm{~km} \mathrm{~s}^{-1}$. We obtained a data cube spanning $10^{\mathrm{h}} 14^{\mathrm{m}} 26^{\mathrm{s}}+21^{\circ} 03^{\prime} 22^{\prime \prime}$ to $10^{\mathrm{h}} 21^{\mathrm{m}} 11^{\mathrm{s}}+23^{\circ} 04^{\prime} 08^{\prime \prime}$ and $700-$ $2010 \mathrm{~km} \mathrm{~s}^{-1}$. The cube has an overall $\mathrm{rms}$ of $2.67 \mathrm{mJy}$ beam ${ }^{-1}$ with a $228 \times 210$ arcsec beam and $5.15 \mathrm{~km} \mathrm{~s}^{-1}$ channels. We note that the data cube was affected by low level RFI in the declination strip that passed through the compact group itself (Leisman et al. 2016), but we are able to take this into consideration with the weights map that is simultaneously created in the ALFALFA data reduction process.

\section{DATA COMBINATION}

Traditional methods of combining radio data between different arrays, or between arrays (interferometers) and singledish measurements, may employ methods which operate in either the $(u, v)$-plane or in the image plane (Stanimirovic 2002 and references therein). The contribution of each measurement is generally weighted by the inverse square of the rms, $1 / \sigma_{r m s}^{2}$. In the case of HCG 44, the WSRT observations are an order of magnitude more sensitive than the KAT-7 data in $\mathrm{Jy} \mathrm{beam}^{-1}$, making an attempt at traditional combination relatively fruitless.

However, recognizing that the WSRT beam is more than an order of magnitude smaller in area than the KAT7 beam, Sorgho et al. (accepted) demonstrate an innovative and powerful method of combining KAT-7 and WSRT mosaics to reveal the presence of a low surface brightness tail in Virgo galaxy, NGC 4424. Their technique realizes the strengths of both telescopes by converting the cubes to column density units which removes the dependence of the measured rms on the beam size, and allows the image cubes to be combined with more equal weights. As a result, the final image of NGC 4424 has the advantage of sensitivity to large-scale structure from the short baselines of KAT-7, and high resolution from WSRT. The full procedure is described in Sorgho et al. (accepted) and we highlight the details relevant to the successful combination of our data below.

We employed the same technique to combine the interferometry data with the ALFALFA maps. The most common method of combining interferometry and single-dish observations is to "feather" the data sets. This refers to performing a Fourier Transform (FT) on the individual data to the $(u, v)$-plane, scaling and combining them, then performing an inverse FT back to the image plane (Stanimirovic 2002). This is typically done because the single-dish and interferometers measure different spatial scales, and the single-dish data is being combined to get the zero-spacing measurement. However, the short baselines of KAT-7 (26-185 m) measure effectively the same spatial scales as Arecibo (illuminated diameter of $208 \mathrm{~m}$ ), and the ALFALFA drift scans have been synthesized to data cubes from which to extract H I detections. Thus, we use the ALFALFA beam information to convert the ALFALFA cube to column density units, and combine with the KAT-7 and WSRT data in the image plane. Formally, as long as the data is weighted appropriately, the methods produce comparable quality results (Stanimirovic 2002).

\subsection{Constructing the final cube}

To optimize the final weighting scheme versus desired resolution, the WSRT cube from Serra et al. (2013) was smoothed with a $90 \times 90$ arcsec Gaussian kernel resulting in an effective restoring beam of $104 \times 95.7$ arcsec and rms noise of $0.39 \mathrm{mJy}$ 


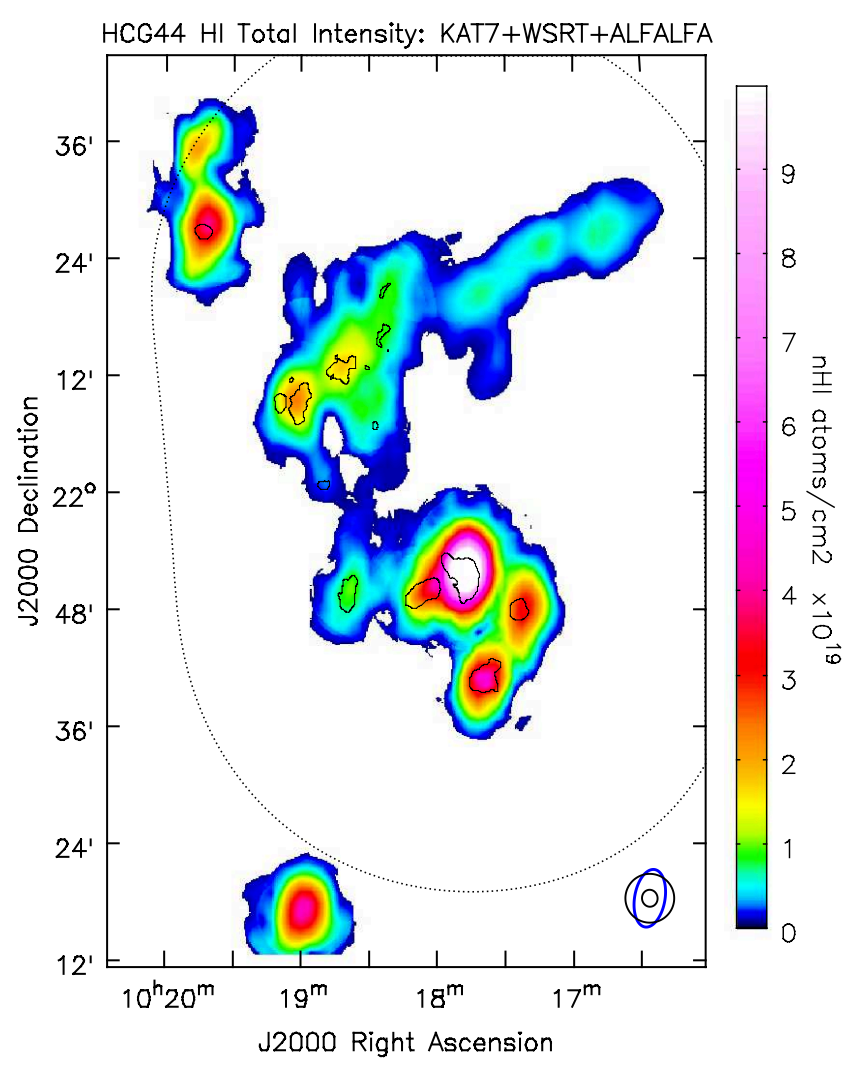

Figure 2. The H I total intensity map of the combined KAT-7, ALFALFA, and WSRT observations (Equation 5). The black contour corresponds to the $1 \times 10^{19} \mathrm{~cm}^{-2}$ column density contours of the original WSRT observation (Serra et al. 2013). The dotted line is the half power of the KAT-7 mosaic. The beams from each telescope are shown in the lower right hand corner, in order of decreasing size: KAT-7 (elongated), ALFALFA (round), smoothed WSRT. The WSRT and ALFLFA data cubes we acquired do not extend as far south as the KAT-7 mosaic, so NGC 3177 does not appear in this image.

beam $^{-1}$. As further preparation, the KAT-7, WSRT, and ALFALFA cubes were smoothed in velocity using a boxcar kernel of 2, 2, and 3 channels, respectively, and decimated in order to achieve the lowest multiple common velocity resolution in independent channels. The KAT-7 and ALFALFA cubes were then regridded to match the velocity and spatial axes of the WSRT cube, so that the resulting cubes have the same pixel size and dimensions $\left(8^{\prime \prime} \times 8^{\prime \prime} \times 16 \mathrm{~km} \mathrm{~s}^{-1}\right)$. All cubes were converted to column density per unit velocity by the following equation:

$N_{H I}=1.1 \times 10^{21}\left(\frac{I_{H I}^{i, j}}{b_{\max } b_{\min }}\right)$

where $I_{H I}^{i, j}$ is the $\mathrm{H}$ I intensity of pixel $(i, j)$ in mJy beam ${ }^{-1} \mathrm{kms}^{-1}$, and $b_{\max }$ and $b_{\min }$ are the major and minor axes of the restoring beam in arcseconds. The resulting KAT-7, WSRT, and ALFALFA data cubes have $\mathrm{rms}$ noise of $1.48 \times 10^{16}, 4.00 \times 10^{16}$, and $3.39 \times 10^{16}$ $\mathrm{cm}^{-2}\left(\mathrm{~km} \mathrm{~s}^{-1}\right)^{-1}$, respectively.

Before linear combination, the KAT-7 and WSRT mosaics were primary beam corrected. CASA automatically generates a primary beam pattern when the visibilities are imaged, which we used to correct the KAT-7 data. With only the final WSRT image cube, we assumed the primary beam correction to be a Gaussian profile with 35 arcmin width. The WSRT data was imaged well beyond the primary beam of the telescope, and if combined linearly with the KAT-7 and ALFALFA data, the final image would be WSRT noise dominated at large distances from the WSRT pointing center. Thus, we only combine with WSRT where the primary beam sensitivity is at least $30 \%$ of the peak.

We experimented with combining the image cubes with increasingly complex set of weights to take into account different considerations. Most simply, the KAT-7 and WSRT primary beam corrected cubes, and ALFALFA cube were weighted by the square of the rms and combined such that:

$I_{\mathrm{KWA}}\left(\mathrm{PB}_{\mathrm{W}}>30 \%\right)=\frac{I_{\mathrm{KAT} 7}+0.14 I_{\mathrm{WSRT}}+0.19 I_{\mathrm{ALFA}}}{1.33}$

$I_{\mathrm{KWA}}\left(\mathrm{PB}_{\mathrm{W}}<30 \%\right)=\frac{I_{\mathrm{KAT} 7}+0.19 I_{\mathrm{ALFA}}}{1.19}$

The resulting cube is dominated by the KAT-7 observations throughout.

Despite the relatively large field of view of KAT-7 (approximately 1 degree at the half power beam width), it also falls off in sensitivity with distance from the mosaic pointing centers, while the ALFALFA sensitivity is fairly flat across the field. Thus, in our final image combination, we take into account not only the relative rms noise at the most sensitive part of the images, but the change in sensitivity across the field of view due to the primary beam (Condon et al. 1998). We also consider the ALFALFA weights map such that:

$I_{\mathrm{KWA}}=\frac{P B_{\mathrm{K}}^{2} I_{\mathrm{K}}+0.14 P B_{\mathrm{W}}^{2} I_{\mathrm{W}}+0.19 w_{\mathrm{A}} I_{\mathrm{A}}}{P B_{\mathrm{K}}^{2}+0.14 P B_{\mathrm{W}}^{2}+0.19 w_{\mathrm{A}}}$.

This cube is dominated by the KAT-7 observations at the center and the ALFALFA observations at the outskirts. This also means that the effective synthesized beam changes across the field. However, because the final beam size of KAT-7 and ALFALFA are similar, we do not consider it to be a significant complication to the interpretation of the final data cube. See the beams in Figure 2, for example. The peak rms sensitivity of this final cube improves to $1.35 \times 10^{16}$ $\mathrm{cm}^{-2}\left(\mathrm{~km} \mathrm{~s}^{-1}\right)^{-1}$ at a velocity resolution of $16.4 \mathrm{~km} \mathrm{~s}^{-1}$. The mean rms across the image is $2.43 \times 10^{16} \mathrm{~cm}^{-2}\left(\mathrm{~km} \mathrm{~s}^{-1}\right)^{-1}$.

Perhaps the greatest benefit of this combination is that the KAT-7 and ALFALFA cubes individually contain residual artifacts whose patterns are characteristic to the telescope, but by combining them one is better able to differentiate between noise and diffuse low-level emission. Throughout the rest of the paper, the $\mathrm{HI}$ data presented are measured or calculated from the combined KAT-7, WSRT, ALFALFA (KWA) image cube, unless otherwise specified (Equation 5).

\section{RESULTS}

In Figure 2, we present the H I total intensity map derived from the combined KWA data cube. The map was made by smoothing the cube with a $5 \times 5$ arcmin beam, and masking regions where the emission was greater than $1.5 \sigma$ in the 
Table 1. Properties of HI Detections

\begin{tabular}{|c|c|c|c|c|c|c|c|c|c|}
\hline Name & $\begin{array}{l}\text { Opt. Pos. } \\
\text { J2000 }\end{array}$ & $\begin{array}{l}\text { Velocity } \\
\mathrm{km} \mathrm{s}^{-1}\end{array}$ & $\begin{array}{l}\text { Dist. } \\
\text { Mpc }\end{array}$ & \multicolumn{2}{|c|}{$\log \left(M_{H I} / M_{\odot}\right)$} & \multicolumn{2}{|c|}{$\log \left(M_{H I} / M_{\odot}\right)$} & \multicolumn{2}{|c|}{$\log \left(M_{H I} / M_{\odot}\right)$} \\
\hline NGC 3185 & $10: 17: 38.5+21: 41: 18$ & 1210 & 25 & - & 8.58 & 8.58 & 8.72 & $8.68^{\dagger}$ & 8.49 \\
\hline NGC $3187^{*}$ & $10: 17: 47.8+21: 52: 24$ & 1575 & 25 & - & & & 9.22 & $9.19^{\dagger}$ & 9.08 \\
\hline NGC $3190^{*}$ & $10: 18: 05.6+21: 49: 56$ & 1300 & 25 & - & 9.23 & 9.25 & 8.82 & $8.88^{\dagger}$ & 8.76 \\
\hline$C_{\mathrm{S}}$ & & 1405 & 25 & - & 8.05 & 8.04 & - & - & 8.08 \\
\hline HCG 44 & & - & 25 & $9.46^{\dagger}$ & 9.34 & 9.35 & 9.45 & $9.44^{\dagger}$ & 9.34 \\
\hline$T_{\mathrm{N}}$ & & $1260-1365$ & 25 & - & 8.89 & 8.88 & - & - & 8.61 \\
\hline Extended Tail & & $1200-1503$ & 25 & - & 9.03 & 9.02 & 9.05 & - & - \\
\hline NGC 3177 & $10: 16: 34.1+21: 07: 23$ & 1295 & 25 & - & 8.90 & - & 8.74 & - & - \\
\hline SDSS J1017 & $10: 17: 23.3+21: 47: 58$ & 1930 & 29 & - & 8.88 & 8.88 & $8.80^{\dagger}$ & - & 8.30 \\
\hline LSBC F567-01 & $10: 19: 01.5+21: 17: 01$ & 1075 & 18 & - & 8.37 & 8.37 & $8.01^{\dagger}$ & - & - \\
\hline UGC 5574 & $10: 19: 42.9+22: 27: 09$ & 1460 & 25 & - & 8.61 & 8.62 & 8.53 & - & $8.12^{\dagger}$ \\
\hline UGC 5575 & $10: 19: 46.8+22: 35: 41$ & 1460 & 25 & - & 8.19 & 8.21 & 8.28 & - & - \\
\hline
\end{tabular}

* NGC 3187 and NGC 3190 are blended in the KAT-7 data. ${ }^{\dagger} \mathrm{H}$ I masses from the literature are corrected to our assumed distance. Where the total H I mass is not reported in the literature, we add the masses from individually reported group members. The telescope columns are listed in order of decreasing beam size. ${ }^{1} 15$ arcmin beam; Borthakur et al. (2015). ${ }^{2} 3.5 \times 3.8$ arcmin beam; Leisman et al.

(2016). ${ }^{3} 60 \times 61$ arcsec beam; we have subtracted HCG 44e from the total H I mass reported for HCG 44 because it is background galaxy at $4032 \mathrm{~km} \mathrm{~s}^{-1}$; Verdes-Montenegro et al. (2001). ${ }^{7} 53 \times 33$ arcsec beam; Serra et al. (2013).

original cube. The mask was inspected and modified to keep regions with emission spatially contiguous over at least 2 channels, and remove peaks suspected to be residual RFI. We took particular care to include even potential emission in the region between HCG 44 and the northern H I tail (see Section 5.2.1 for further explanation). The mask was similarly inspected and applied to the KAT-7 data and was used to generate the contours overlaid in Figure 1.

The addition of the KAT-7 and ALFALFA observations shows that the H I tail extends further than previously detected with WSRT. Based on low signal-to-noise HIPASS images, Serra et al. (2013) found that the tail stretched 300 $\mathrm{kpc}$ in projection. At the assumed distance of $25 \mathrm{Mpc}$ (see Section 4.1), we find the extended tail spans nearly $450 \mathrm{kpc}$ (62 arcmin) in projection and is arguably resolved into seven to eight clumps. The extent is measured from the southern extension (cloud 1), through the brightest part of the tail, to the northwestern cloud 8 . The physical length of the tail may be even longer than what is seen in projection. Our images reveal the breadth, mass, column density, and velocity dispersion of the tail that was unresolved by HIPASS. Throughout the rest of this work, we will refer to the tail resolved by the WSRT maps as $T_{\mathrm{N}}$, and the full extent revealed by the KWA combined data, which includes $T_{\mathrm{N}}$, as the "extended tail."

In addition to HCG 44, we detect the suspected background galaxy, SDSS 1017723.29+214757.9 (hereafter SDSS 1017; Serra et al. 2013), and likely foreground galaxy, LSBC F567-01. We note that the elliptical galaxy, NGC 3193, remains undetected in H I. Aguerri et al. (2006) propose that despite, its velocity $\left(1381 \mathrm{~km} \mathrm{~s}^{-1}\right)$, the absence of planetary nebulae in narrow band observations suggest it lies behind the compact group. Finally, UGC 5574 and UGC 5575 are also detected and, while not formally part of HCG 44, are likely part of the larger associated group of galaxies (Makarov \& Karachentsev 2011).

We present the properties of all the $\mathrm{HI}$ detections in Table 1 with comparison to previous detections. In order to convert from column density to solar masses we use
$1 \mathrm{M}_{\odot} \mathrm{pc}^{-2}=1.248 \times 10^{20}$ atoms $\mathrm{cm}^{-2}$, integrate over the volume of the emission in the cube, and use the pixel scale at the luminosity distance of the object to convert to parsec ${ }^{2}$. For the literature values, we take the published integrated flux and convert to H I mass using our assumed distances, and the standard equation:

$M_{H I} / M_{\odot}=2.36 \times 10^{5} D_{\mathrm{Mpc}}^{2} \int S_{21} d v$

where $\int S_{21} d v$ is the intergrated flux in $\mathrm{Jy} \mathrm{km} \mathrm{s}^{-1}, D_{\mathrm{Mpc}}$ is the distance in $\mathrm{Mpc}$, and $M_{\mathrm{H} \text { I }}$ is the H I mass computed in units of solar mass. These values are given in Table 1 .

\subsection{A note on distances}

The greatest source of error on the true Hi mass content is the uncertainty in distance to individual galaxies both in and around the compact group. NED gives estimates corrected for Virgo infall of order $22-25 \pm 2 \mathrm{Mpc}$, while distances assumed in the literature range from 18.4 to 25 Mpc (Verdes-Montenegro et al. 2001; Borthakur et al. 2015; Williams et al. 1991; Serra et al. 2013). The NGC 3190 group itself is part of a broader filamentary structure, so distance estimates from recessional velocities can be significantly distorted by infall onto the filament. Following the convention of WSRT and ALFALFA studies, we assume a distance of $25 \mathrm{Mpc}$ to HCG 44 member galaxies NGC 3185, NGC 3187, NGC 3190, to the cloud, $C_{\mathrm{S}}$, and the extended tail. We assume the same for NGC 3177, UGC 5574, and UGC 5575 which have similar recessional velocities and are at the same distance, within uncertainties, in velocity flow models of the region (see Table 1). Leisman et al. (2016)) present a detailed discussion of distances to galaxies in the region.

\subsection{KAT-7 versus ALFALFA masses}

Table 1 provides a comparison of the H I masses between the KAT-7 observations, the KWA results, and previous obser- 
vations from a range of telescope facilities. A cursory look reveals that the interpretation of fluxes from different facilities is not straight forward. The amount of $\mathrm{H}$ I detected by a given telescope is dependent on the size scales to which it is sensitive. For example, if there is a significant amount of intragroup gas, we expect KAT-7 and ALFALFA to report more gas than WSRT, perhaps less gas than the GBT, and produce similar results to one another. Errors in the absolute flux calibration would also produce different results between facilities. Recalibrating data from five different telescopes to ensure consistency in the absolute flux calibration is outside the scope of this paper, however our observations give us the opportunity to comment on the relative flux calibration between KAT-7 and ALFALFA.

Among systematic sources of error, fitting a baseline to the data is particularly critical to account for both broadband continuum emission, and small scale residual bandpass fluctuations that may remain to separate the spectral line emission of individual galaxies (van Zee et al. 1997). For both KAT-7 and ALFALFA the baseline fitting takes place in a two step process. There is a first pass to remove broad continuum emission, and a second pass when the detections are measured which fits for local fluctuations of the baseline in emission free channels over the region in which the spectral line flux is extracted.

Springob et al. (2005) show that H I flux densities measurements are typically not better than $15 \%$. The mean difference between KAT-7 and ALFALFA H I masses is $21 \%$. However, there is not a simple universal offset. Generally, the galaxies at the center of the KAT-7 mosaic (HCG 44 members and SDSS J1017) are underestimated in the KAT7 data compared to ALFALFA, and the galaxies on the outskirts of the mosaic (NGC 3177, LSBC F567-01, and UGC 5574), where the sensitivity of the KAT-7 data are declining, are overestimated.

One possible explanation is that a better primary beam model is required for KAT-7 than the Gaussian assumed by the CASA software. A full model for the KAT-7 primary beam is not currently available, but holography measurements of the individual dishes were taken as part of the commissioning process. In Figure 3 we present the amplitude beam measurement of Antenna 1 Stokes I for KAT-7 compared to the Gaussian primary beam used to correct the data. The engineering holography measurement has been arbitrarily scaled in the radial direction for comparison. We see that, indeed, it appears the Gaussian will under correct flux close to the center of a pointing, and over correct the flux on the outskirts. For the Antenna 1 measurement, this difference is at the $2 \%$ level, but it may be greater when the entire array is considered together.

\section{DISCUSSION}

A study of 22 HCGs, which included HCG 44, provided evidence of a diffuse neutral gas intragroup medium present in some compact groups (Borthakur et al. 2010). However, based on follow-up mapping with the GBT, it was argued from the strong double-horned H I profiles that the majority of $\mathrm{HI}$ in HCG 44 is bound in stable disk-like structures (Borthakur et al. 2015). These observations covered the three main galaxies in HCG 44: NGC 3185, NGC 3187,

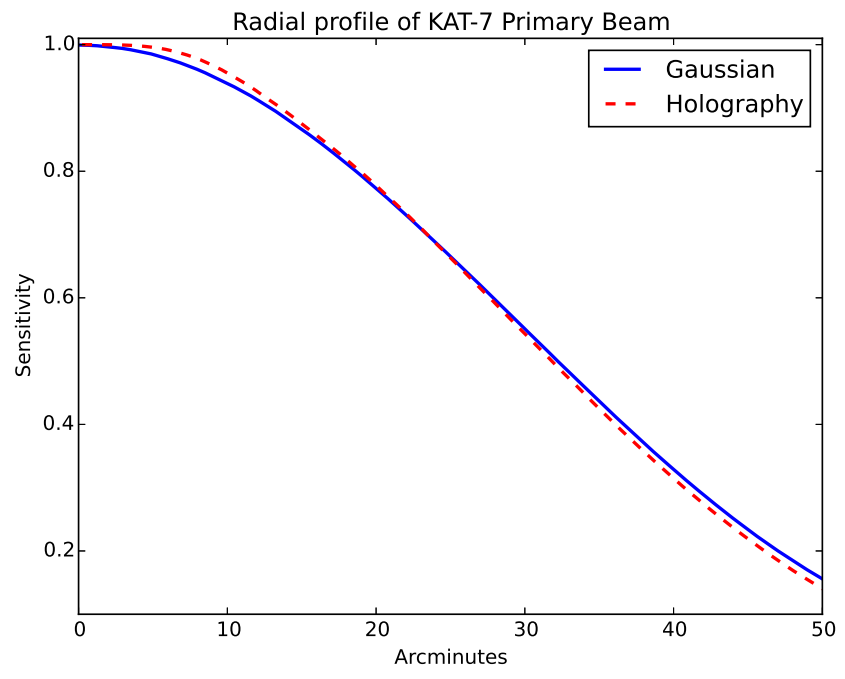

Figure 3. A comparison of the assumed Gaussian primary beam used to correct the KAT-7 data (solid blue line) with engineering holography measurements of Antenna 1 Stokes I amplitude (dashed red line). The holography data has been scaled radially to illustrate the approximate difference in primary beam correction which could account for the pattern of HI flux measurements. The greatest difference for Antenna 1 is approximately $2 \%$, but may be greater for a full KAT-7 primary beam model.

NGC 3190; and the nearby $C_{S}$ cloud, and found a total H I mass of $2.9 \times 10^{9} \mathrm{M}_{\odot}$ but did not resolve the compact group. We measure $2.3 \times 10^{9} \mathrm{M}_{\odot}$ in HCG 44 , which is consistent with the total H I mass resolved by WSRT Serra et al. (2013). While the GBT observations appear to detect $22 \%$ more H I in HCG 44 than either the KWA or WSRT observations, this seems to be within the variation measured for individual galaxies by different telescopes, even when those telescopes are sensitive to the same spatial scales. The GBT and ALFALFA values are also consistent with the VLA-D observations, which show the $\mathrm{HI}$ is confined to individual galaxies (Williams et al. 1991). Thus, we conclude that even with deeper $\mathrm{H}$ I observations, there is no strong evidence for a diffuse, neutral IGM in HCG 44.

\subsection{H I Deficiency in HCG 44}

HCG 44 is known to be highly Hi deficient, even when compared to other compact groups. The expected H I mass for the group is calculated by summing the predicted values from the individual galaxies based on their morphological type (Haynes \& Giovanelli 1984) and comparing them to the sum of the observed Hi masses: $D e f_{H I}=\log \left[\Sigma M(H I)_{\text {pred }}\right]-\log \left[\Sigma M(H I)_{\text {obs }}\right]$. The predicted value for HCG 44 is $\log \left[\Sigma M(H I)_{\text {pred }}\right]=10.2 \pm 0.2$ (Verdes-Montenegro et al. 2001). Our observed value is in line with previous measurements: $\log \left[\Sigma M(H I)_{\text {obs }}\right]=9.35 \pm$ 0.2 .

Verdes-Montenegro et al. (2001) point out that the deficiency in HCG 44 is not due to a few highly anemic objects, but is a characteristic shared by all the members. This argues that the group members have evolved together to account for the high H I deficiency, and against a single interloper which has had its gas completely removed. The most 
probable effect produced by tidal interactions is believed to be disk stripping (Verdes-Montenegro et al. 2001). The role of ram-pressure stripping in compact groups has been shown to be small, only capable of removing small amounts of cold gas (Rasmussen et al. 2008; Freeland et al. 2010), however, turbulent viscous stripping due to hydrodynamical interactions may also be an important mechanism for removing gas from compact group members (Nulsen 1982; Quilis, Moore \& Bower 2000).

\subsection{The Extended $\mathrm{H}$ I tail}

The advantage of low surface brightness sensitivity and wide field of view of our observations are apparent in observing the $\mathrm{HI}$ tail, $T_{\mathrm{N}}$, where we find $\sim 90 \%$ more $\mathrm{HI}_{\mathrm{I}}$ than the WSRT observations alone. However, the most striking result of our observations is detecting and resolving the gas beyond $T_{\mathrm{N}}$. The extended tail is resolved into 7-8 distinct knots, and the total H I mass is of the order observed in a typical galaxy: $1.1 \times 10^{9} \mathrm{M}_{\odot}$. Nonetheless, even including the extended tail, the total gas content of the HCG 44 system only adds up to $\log \left[\Sigma M(H I)_{\text {obs }} / M_{\odot}\right]=9.52 \pm 0.1$ and cannot account for the observed H I depletion of the compact group.

Figure 4 shows the intensity weighted velocity map of the extended $\mathrm{H}$ I tail. The extended tail is contiguous in velocity with a shallow gradient that is parallel with the long axis of the tail from $\sim 1365-1245 \mathrm{~km} \mathrm{~s}^{-1}$. In fact, the westernmost extent, at $1270 \mathrm{~km} \mathrm{~s}^{-1}$, is not as blueshifted as the center of the tail at $1250 \mathrm{~km} \mathrm{~s}^{-1}$. Meanwhile the most massive clumps in the tail, which increase in mass to the east, are continuous from $1260-1365 \mathrm{~km} \mathrm{~s}^{-1}$. Figure 5 shows the extended tail forms a shallow arc in velocity. Despite the 350 kpc extent of tail, it is quite narrow in velocity with a dispersion of only $\sim 25-55 \mathrm{~km} \mathrm{~s}^{-1}$. In the most massive clumps, $T_{\mathrm{N}}$ has a dispersion of roughly $45-55 \mathrm{~km} \mathrm{~s}^{-1}$, which peaks in cloud 3 . Beyond $T_{\mathrm{N}}$, clouds 6,7 , and 8 have a dispersion of $25-35 \mathrm{~km} \mathrm{~s}^{-1}$.

\subsubsection{Tail origins?}

While $\mathrm{H}$ I is invaluable for recognizing a history of past interactions, disentangling the chain of events is more complicated. The origin of the extended $\mathrm{H}$ I tail associated with HCG 44 is no less mysterious. Serra et al. (2013) ruled out the likelihood that the tail is the result of ram pressure from the lack of diffuse X-ray emission. The authors discussed two possible scenarios. First, the gas was pulled out from NGC 3162 in a close passage with the compact group. The elongated morphology of $T_{\mathrm{N}}$ and the HIPASS detection suggest a tenuous connection between the tail and NGC 3162, which lies approximately $620 \mathrm{kpc}$ northwest of HCG 44 and the same distance from cloud 2 of the tail. Second, the gas originated from one of the galaxies currently within HCG 44 that has interacted with the group gravitational potential. Within HCG 44 both the stellar and resolved H I component of NGC 3187 show a strong S-shaped distortion (Figure 1 and the WSRT contours in Figure 2) suggesting a strong tidal interaction. Meanwhile, in SDSS optical images NGC 3190 has a strong dust lane with a swept-back morphology looking as though it has passed from the direction of $T_{\mathrm{N}}$, and the galaxy has strong infrared $\mathrm{H}_{2}$ emission which has been attributed to shocked gas (Cluver et al. 2013).

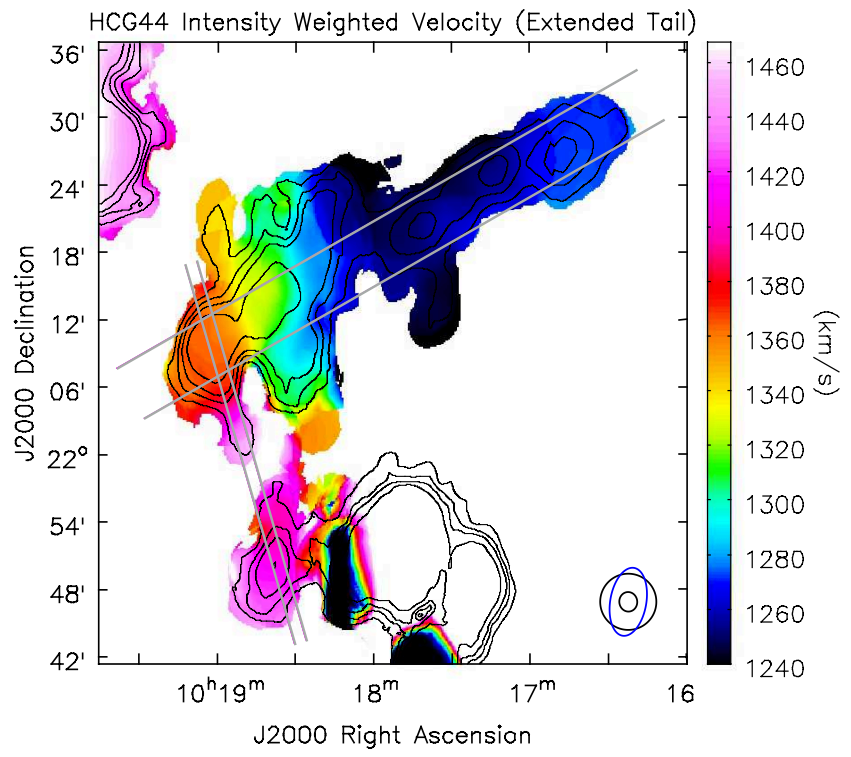

Figure 4. The intensity-weighted velocity map of the $\mathrm{H}$ I tail. Although the tail has distinct peaks in intensity, it is continuous in velocity along the full length. Contours are at 2, 4, 6, $10 \times$ $10^{18} \mathrm{~cm}^{-2}$. Parallel gray lines illustrate the orientation and width of the position-velocity slices in Figure 5 and 6 . The beams for individual observations are shown in the lower right hand corner as in Figure 2.

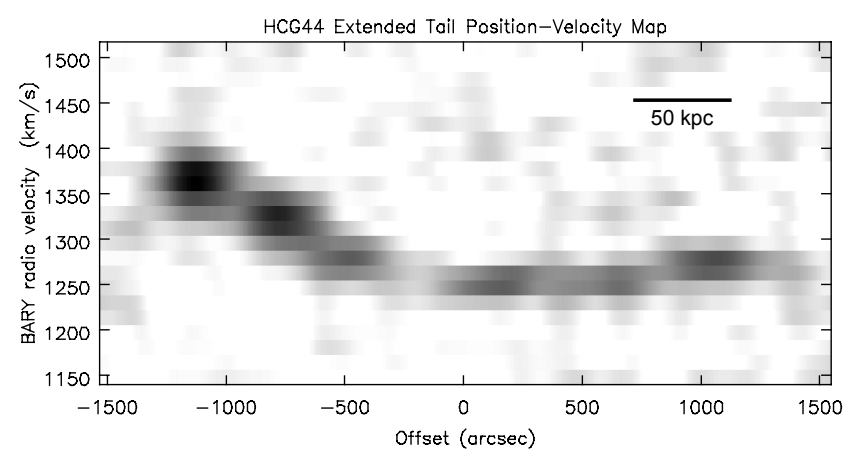

Figure 5. A position-velocity slice taken along the main body of the $\mathrm{H}$ I tail. The data is summed over a slice 5.2 arcmins wide that cover the major clouds within the tail. The velocity spans $\sim 120$ $150 \mathrm{~km} \mathrm{~s}^{-1}$, and the dispersion is of order $25-55 \mathrm{~km} \mathrm{~s}^{-1}$ across the full $350 \mathrm{kpc}$ length of the tail.

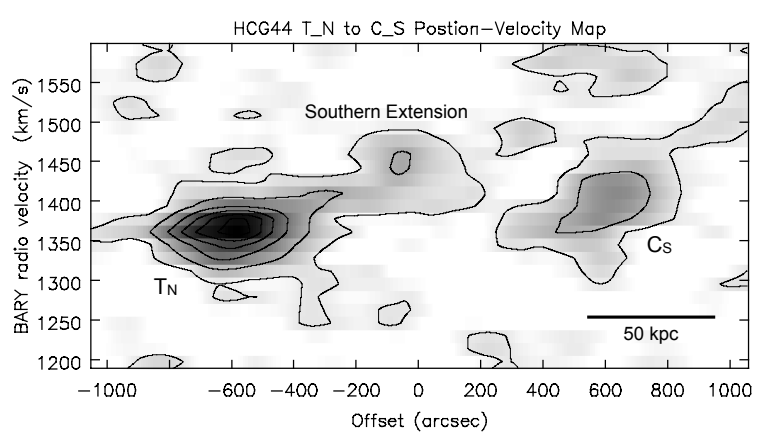

Figure 6. A position-velocity slice taken between $T_{\mathrm{N}}$ and $C_{\mathrm{S}}$. The data is summed over a slice 1.2 arcmins wide. The contours have arbitrary values and are included to guide the eye. There is no strong evidence for an HI bridge to the compact group beyond the southern extension of $T_{\mathrm{N}}$.

MNRAS 000, 1-12 (2016) 
An outstanding question has been whether Serra et al. (2013) catch a glimpse of the high column density gas in an $\mathrm{H}$ I bridge which connects $T_{\mathrm{N}}$ to the compact group. If such a bridge exists, it should be visible in both our KAT-7 and combined images in the most sensitive region of the cubes. A connection would add weight to the second origin scenario, and we could trace from which HCG 44 member galaxy the gas was stripped. We went through a concerted effort to uncover such a bridge, going through multiple iterations of targeted cleaning of the KAT-7 cube during the imaging process, inspecting cubes at all stages of post-processing, including the KAT-7 cube, the final data cube described in Section 3, the spatially smoothed cube used for masking, and previous versions of the cubes at higher velocity resolution. Finally we inspected position-velocity slices of the cubes at various angles from NGC 3190 and NGC 3187 towards the high column density clumps of $T_{\mathrm{N}}$. Ultimately, we confirm faint continuous emission between cloud 1 and cloud 2 of $T_{\mathrm{N}}$, which makes up the southern extension, but we found no convincing evidence for an $\mathrm{H}$ I bridge connecting $T_{\mathrm{N}}$ to any of the HCG 44 members at our detection limits (e.g. Figure $6)$.

The gap in $\mathrm{H}$ I emission between $T_{\mathrm{N}}$ and HCG 44 may be explained if the $\mathrm{H}_{2}$ emission in NGC 3190 is not the result of shocked gas due to collisions with tidal debris, but comes from the presence of an active galactic nucleus (AGN; Martínez et al. 2010) due to jet-interstellar medium (ISM) interactions (Ogle et al. 2010). The orientation of NGC 3190 is such that ionizing radiation emanating from the nucleus would have a clear path in the direction of where a bridge between HCG 44 and $T_{\mathrm{N}}$ would exist. Thus, it is possible that we do not detect an $\mathrm{H}$ I bridge because it has been blown out or ionized, similar to gaps seen in $\mathrm{H}$ I tidal features such as around NGC 520 (Hibbard et al. 2000).

On the other hand, images from ALFALFA which cover a larger area of sky show increasing evidence for a connection between the extended tail and NGC 3162 (Leisman et al. 2016). It is not obvious that the gas must have originated from NGC 3162 because there is still a gap in the observed H I distribution between the tail and NGC 3162. However, we note that the morphology of the extended tail is consistent with that of gas pulled out of galaxies in other high velocity encounters $(v \sim 1000 \mathrm{kms})$, such as VIRGOHI 21 near NGC 4254 (Duc \& Bournaud 2008). In these simulations, the $\mathrm{H}$ I tidal tails that form are long, narrow, and have a low stellar content.

There are further considerations, although it is unclear as to how they should be interpreted. First, the tail is resolved into clumps and does not have a large velocity gradient as is seen in the Duc \& Bournaud (2008) simulations, although the latter may be projection effects. Second, the most massive H I clouds are those furthest from NGC 3162, and closest to HCG 44. The most massive clouds to result from a tidal interaction are likely those pulled out near periastron, where tidal forces are the greatest. Third, the tail is the messiest to the southeast, close to HCG 44. This could be because the gas originated within the compact group, or if the tidal tail was pulled out from NGC 3162 it is now interacting with the compact group. Finally, we note that NGC 3162 does not suffer from H I deficiency (Leisman et al. 2016), although the uncertainty in expected H I mass for a galaxy of a given morphology and diameter is enough that we cannot rule out NGC 3162 as the donor.

In any case, it is probable that multiple interactions have occurred in the system. A third scenario is that multiple interactions within the compact group loosened the cold gas of the galactic ISM and allowed it to form a long tail in a high speed encounter with NGC 3162, as modeled by Duc \& Bournaud (2008). The internal interactions shake up the H I gas which is then not bound as tightly in the shallow group potential, and would also account for the disturbed morphology of NGC 3190. The overall H I deficiency of galaxies in the compact group would be the result of their history of group membership and gas depletion through star formation and gas processing, as proposed in other group environments by Verdes-Montenegro et al. (2001) or Hess \& Wilcots (2013). If the gas in the extended tail originated in an HCG 44 galaxy, it has not had to travel as far from its starting point as if it originated in NGC 3162. In short, we present a number of possible scenarios for the origin of the extended tail, but the KWA data presented here is unable to say conclusively which is correct.

\subsubsection{Collapse or Dispersal?}

Despite the uncertainty in its origin, we investigate the possible fate of the H I tail. Our data shows that it has a clumpy distribution with 7-8 knots of emission. If we consider each knot as a distinct $\mathrm{H}$ I cloud, we note that the clouds with the highest peak column density and greatest velocity dispersion are the easternmost clumps of the tail.

Tidal interactions are known to sometimes produce knots of star formation in stripped gas, and if these structures are self-gravitating, they are referred to as tidal dwarf galaxies (Barnes \& Hernquist 1992; Duc \& Mirabel 1999). Assuming the clouds are spherical, and the observed velocity dispersion is the thermal velocity of the gas, we can test to see if they will collapse. The easternmost knot is $2.2 \times 10^{8} \mathrm{M}_{\odot}$ and has a velocity dispersion of $\sim 50 \mathrm{~km} \mathrm{~s}^{-1}$. The Jeans' length is $R=3 / 5 G M / \sigma^{2} \approx 0.2 \mathrm{kpc}$. The WSRT appears to resolve this cloud to the size of 5 arcminutes which corresponds to $36 \mathrm{kpc}$ (Figure 2, or Figure 2 in Serra et al. 2013) so it is unlikely that stars are forming out of gas in the tail. Additionally, despite deep images from CFHT/MegaCam with a limiting surface brightness sensitivity of $\sim 28.5 \mathrm{mag} \operatorname{arcsec}^{-2}$ in g-band (Serra et al. 2013), there is no known stellar component in the optical, and nothing evident in WISE infrared imaging (Figure 1). If stars were pulled out of the galaxy concurrently with the gas, they would be broadly dispersed and potentially undetectable (Hibbard et al. 2000). Searches for planetary nebula line emission as a tracer for intragroup stellar light, conclude an upper limit of only $5 \%$ for the diffuse light fraction within HCG 44 itself (Aguerri et al. 2006).

On the other hand, we may investigate cloud survival in the context of photoionization. Observations have shown that both the outer disks of galaxies and tidal debris are protected from the ionizing ultraviolet (UV) background if the gas is above a few $\times 10^{19} \mathrm{~cm}^{-2}$ (Corbelli et al. 1989; van Gorkom 1991; Hibbard et al. 2000). Radiative transfer modeling suggests that clouds can survive in the intergalactic medium if they have a column density of at least $2 \times 10^{19}$ $\mathrm{cm}^{-2}$ (Maloney 1993; Borthakur et al. 2015). In our total 
intensity map (Figure 2) the extended envelope of gas in the $\mathrm{HI}$ tail is closer to $1 \times 10^{19} \mathrm{~cm}^{-2}$ or less, but this may be an underestimate since the clouds themselves are largely unresolved. The peak column density of clouds 2 and 3 are $2.3 \times 10^{19}$ and $1.8 \times 10^{19} \mathrm{~cm}^{-2}$, respectively.

Borthakur et al. (2015) point out that a $1 \mathrm{kpc}$ cloud with an ISM density of 1 atom $\mathrm{cm}^{-3}$ expanding at $20 \mathrm{~km} \mathrm{~s}^{-1}$ would survive for $\sim 500$ Myr before dropping to a density susceptible to photoionization by the UV background. However, if the density of the IGM is of order a few to tens $\times 10^{-4} \mathrm{~cm}^{-3}$ the cold $\mathrm{H}$ I clouds will reach pressure equilibrium before reaching the critical column density, and may survive longer. These IGM densities have been inferred indirectly through the morphology of bent jets in galaxy groups (Freeland \& Wilcots 2011).

We find that both the column density and velocity dispersion of the $\mathrm{H}_{\mathrm{I}}$ clouds decrease as one moves along the tail from east to west (see Figure 5 and Section 5.2). If we consider that the gas has been pulled out of NGC 3187, as in Serra et al. (2013)'s 3D toy model, and/or NGC 3190, the clouds may represent snapshots of evolution at different times after they have been pulled out of the galaxy. In this case the $\mathrm{H}$ I clouds become fainter as they disperse and the velocity dispersion decreases as what remains is the cold core of the cloud. Our extrapolation of the proposed trajectory from the 3D model implies that the easternmost $\mathrm{H}$ I clouds were stripped of order 500, 600, and 700 Myr ago. Further, the clouds in the extended tail fall at intervals which would imply they were stripped approximately 850, 1000, and $1100 \mathrm{Myr}$ in the past. These lifetimes suggest that intragroup $\mathrm{H}$ I clouds may have survived by being contained in pressure equilibrium with a warm intragroup medium that has not been directly detected, and which may extend out to quite large distances beyond the compact group-perhaps as part of the larger GH58 or NGC 3190 loose group to which HCG 44 apparently belongs (Geller \& Huchra 1983; van Driel et al. 2001; Makarov \& Karachentsev 2011).

Alternatively, we can make a simple estimate of the required survival time of the clouds if they are a result of a close encounter with NGC 3162. NGC 3162 lies at a projected distance of $620 \mathrm{kpc}$ from $\mathrm{HCG} \mathrm{44}$, and at relative fly-by velocities of $600-200 \mathrm{~km} \mathrm{~s}^{-1}$, the clouds would have had to survive between 1-3 Gyr. The lower velocity limit seems challenging, given the long required lifetime of the clouds in the IGM. Meanwhile, the high velocity limit has been shown to produce long tidal tails in cluster systems, but may be unusually high for a galaxy group system with few members and an otherwise narrow velocity dispersion (115-220 $\mathrm{km} \mathrm{s}^{-1}$ depending on what is considered part of the group; Leisman et al. 2016, Geller \& Huchra 1983).

Simulations of small $\mathrm{H}$ i clouds survival in a Milky Waylike hot $\left(T=1-3 \times 10^{6} \mathrm{~K} ; n_{H} \approx 1-3 \times 10^{-4} \mathrm{~cm}^{-3}\right)$ halo suggest that neutral $\mathrm{HI}$ which has been stripped can last of order 80-200 Myr before it is disrupted or ionized (Fernández et al. 2012; Heitsch \& Putman 2009). The H I clouds of these simulations have a range of mass from $\left(\sim 10^{4-8} \mathrm{M}_{\odot}\right)$, but Putman et al. (2012) summarizes that total mass seems to be one of the largest factors which increase their lifetime. The hydrodynamic simulations also include the effects of ram pressure stripping, in addition to photoionization. If the effects of ram pressure are less severe on the HCG 44 intragroup clouds (Hibbard et al. 2000), per- haps they are able to live longer. If they have already lived in the IGM for 1 Gyr, they probably started with a larger initial mass than what we currently observe.

\subsection{H I in Group Evolution}

$\mathrm{H} \mathrm{I}$ is a key tracer of physical mechanisms at work in galaxy groups as the gas is both the most extended component of pristine galaxies, and also most easily removed through gravitational and hydrodynamical interactions. It can provide rigorous constraints on galaxy-galaxy interactions, group dynamics, and indirect evidence of the warm-hot IGM which remains otherwise undetected. Tidally stripped H I in groups appears to survive as both filamentary structures and a diffuse component (Verdes-Montenegro et al. 2001; Borthakur et al. 2010). This gas can provide a reservoir for re-accretion through so-called "rejuvenation events", but collisions with this intragroup medium may also produce shocks in the interstellar medium of galaxies (Cluver et al. 2010, 2013). Associated shock heating may lead to further stripping, or the formation of molecular hydrogen through gas compression at the leading edge of the shock(Appleton et al. 2006; Cluver et al. 2013). To date the preponderance and evolutionary effects of these processes remain relatively unexplored and poorly understood. In HCG 44, if previously detected $\mathrm{H}_{2}$ is the result of shocks due to collisions with the IGM, the intragroup medium doing the work remains undetected in our deep $\mathrm{H}$ I data. On the other hand, if the $\mathrm{H}_{2}$ emission is due to jet-ISM interaction shocks from an AGN in NGC 3190, it may explain the absence of an $\mathrm{H}$ i bridge between HCG 44 and the extended H I tail.

Qualitative analytical arguments for $\mathrm{H}$ I cloud survival have estimated cold cloud lifetimes of 500 Myr once gas has been removed from a galaxy disk Borthakur et al. (2015). However, if the orbit of NGC 3187 modeled by Serra et al. (2013) is representative of a group member interacting with the HCG 44 group potential, the H I clouds at the end of the extended tail may have already survived for up to $1 \mathrm{Gyr}$. If the gas originated in NGC 3162, the clouds have lived in the circumgroup medium for 1-3 Gyr. This result warrants further simulation to understand how the balance of physical processes between the cloud and the circumgroup medium allows for cloud survival. Unfortunately, the warm-hot intragroup medium has thus far evaded direct detection so the density and temperature of the intragroup medium is constrained more by what we do not see than what we do (e.g. Davé et al. 2001; Freeland \& Wilcots 2011). However, our result demonstrates the potential of $\mathrm{H}$ i to provide even stronger constraints on the modeling of galaxy group properties.

Future wide-area H i surveys (e.g. WALLABY, DINGO, MIGHTEE-HI, and APERTIF surveys) will uncover many more nearby $\mathrm{H}$ I rich groups for deep follow-up. A number of preliminary results from deep KAT-7 observations (Oosterloo et al, in prep; Lucero, private communication) and from ASKAP (Serra et al. 2015), show that extended and lowsurface brightness H i features may be even more common in galaxy groups than previously realized. Tidal interactions produce material that lead to viscous stripping of the interstellar medium from galaxies, and this process may be important in loose groups in addition to compact groups.

Finally, with the expected increase in the gas fraction of 
galaxies as one moves to higher redshift, group interactions may play an even more significant role in the evolution of galaxies in the past than what is seen locally. In other words, the effect of environment when galaxies were typically gasrich $(z \sim 1-2)$ may be a key consideration at a time when the universe was experiencing elevated star formation and more efficiently building stellar mass compared to local systems.

\section{CONCLUSIONS}

We observed the region around HCG 44 and the giant H I tail discovered by Serra et al. (2013), which extends to the north and west of the compact group, to investigate the extent of intragroup H I. We combined a deep two-pointing mosaic from KAT-7 with imaging from the ALFALFA survey, and a single pointing observation of WSRT. Despite the very different nature of the telescopes, we successfully combined the data by converting the image units of $\mathrm{Jy} \mathrm{beam}^{-1}$ to column density per unit velocity, atoms $\mathrm{cm}^{-2}\left(\mathrm{~km} \mathrm{~s}^{-1}\right)^{-1}$, weighting by the sensitivity of the respective observations, and adding them in the image plane.

We find no evidence for copious amounts of neutral $\mathrm{H}$ I in the IGM within the core of the compact group, which suggests the $\mathrm{H}_{2}$ emission observed by Cluver et al. (2013) in NGC 3190 may be the result of AGN activity and jetISM interaction rather than shocked gas from NGC 3190 plunging through the IGM. The presence of an AGN, or star formation in the nucleus of NGC 3190 could account for the lack of an Hi bridge between HCG 44 and the H I tail, if HCG 44 is where the extended H I tail originated from.

The combined H I data show that the H I tail extends out to at least $450 \mathrm{kpc}$ and is resolved into $7-8$ clouds at the resolution of KAT-7. We find more $\mathrm{H}$ I mass in all parts of the tail as compared to previous WSRT observations, such that the total $\mathrm{H}$ I mass in the tail is $1.1 \times 10^{9} \mathrm{M}_{\odot}$. However, the gas in the tail is still not able to account for the overall $\mathrm{H}$ I depletion in the compact group. The remaining gas is either too diffuse to be measured with current facilities, or more likely has been ionized and may exist in a warm-hot group halo that is also undetected in X-rays.

We find that if the gas in the tail is stripped from one of the HCG 44 member galaxies, and the timescale for stripping proposed in Serra et al. (2013) is correct, the gas must have been able to live in the intragroup medium without being ionized for 0.5-1 Gyr, which is longer than previously predicted. If the gas originated in NGC 3162, it must have been produced in a high speed encounter, and even then must have survived in the IGM for well more than 1 Gyr. Regardless of the origins of the $\mathrm{H}$ I tail, our results demonstrate indirect evidence for a surrounding medium which has confined the expansion of the $\mathrm{H}$ I clouds and prevented their destruction by background UV radiation. Targeted simulations are required to explain how these $\mathrm{H}$ I clouds can survive for long periods of time in the IGM.

\section{ACKNOWLEDGEMENTS}

The research of KMH has been supported by the European Research Council under the European Union's Sev- enth Framework Programme (FP/2007-2013)/ERC Grant Agreement nr. 291531. MEC acknowledges support from the South African National Research Foundation. The work of SY was supported by the 2015 ASTRON/JIVE Summer Student Programme. The work of CC is based upon research supported by the South African Research Chairs Initiative (SARChI) of the Department of Science and Technology (DST), the Square Kilometre Array South Africa (SKA SA) and the National Research Foundation (NRF). We thank Thijs van der Hulst, DJ Pisano, Lourdes Verdes-Montenegro for useful discussions.

This work is based on observations obtained at the KAT-7 array which is operated by the SKA SA on behalf of the National Research Foundation of South Africa.

The authors would like to acknowledge the work of the entire ALFALFA collaboration team in observing, flagging, and generating the data products used in this work. The ALFALFA team at Cornell is supported by NSF grants AST0607007 and AST-1107390 to RG and MPH and by grants from the Brinson Foundation.

This paper made use of observations obtained with the Westerbork Synthesis Radio Telescope, which is operated by the ASTRON (Netherlands Foundation for Research in Astronomy) with support from the Netherlands Foundation for Scientific Research (NWO).

This publication makes use of data products from the Wide-field Infrared Survey Explorer, which is a joint project of the University of California, Los Angeles, and the Jet Propulsion Laboratory/California Institute of Technology, funded by the National Aeronautics and Space Administration (NASA).

\section{REFERENCES}

Abazajian, K. N., Adelman-McCarthy, J. K., Agüeros, M. A., et al. 2009, ApJS, 182, 543

Aguerri, J. A. L., Castro-Rodríguez, N., Napolitano, N., Arnaboldi, M., \& Gerhard, O. 2006, A\&A, 457, 771

Appleton, P. N., Xu, K. C., Reach, W., et al. 2006, ApJ, 639, L51

Barnes, D. G., \& Webster, R. L. 2001, MNRAS, 324, 859

Barnes, J. E., \& Hernquist, L. 1992, Nature, 360, 715

Berlind, A. A., Frieman, J., Weinberg, D. H., et al. 2006, ApJS, 167, 1

Berlind, A. A., \& SDSS 2009, Bulletin of the American Astronomical Society, 41, 425.01

Bicay, M. D., \& Giovanelli, R. 1986, AJ, 91, 705

Borthakur, S., Yun, M., \& Verdes-Montenegro, L. 2010, ApJ, 710, 385

Borthakur, S., Yun, M. S., Verdes-Montenegro, L., et al. 2015, ApJ, 812, 78

Carignan, C., Frank, B. S., Hess, K. M., et al. 2013, AJ, 146, 48

Cluver, M. E., Appleton, P. N., Boulanger, F., et al. 2010, ApJ, 710,248

Cluver, M. E., Appleton, P. N., Ogle, P., et al. 2013, ApJ, 765, 93

Condon, J. J., Cotton, W. D., Greisen, E. W., et al. 1998, AJ, 115,1693

Corbelli, E., Schneider, S. E., \& Salpeter, E. E. 1989, AJ, 97, 390

Davé, R., Cen, R., Ostriker, J. P., et al. 2001, ApJ, 552, 473

Desjardins, T. D., Gallagher, S. C., Tzanavaris, P., et al. 2012, arXiv:1212.1151

Driver, S. P., Norberg, P., Baldry, I. K., et al. 2009, Astronomy and Geophysics, 50, 12 
Duc, P.-A., \& Mirabel, I. F. 1999, Galaxy Interactions at Low and High Redshift, 186, 61

Duc, P.-A., \& Bournaud, F. 2008, ApJ, 673, 787-797

Fernández, X., Joung, M. R., \& Putman, M. E. 2012, ApJ, 749, 181

Freeland, E., Sengupta, C., \& Croston, J. H. 2010, MNRAS, 409, 1518

Freeland, E., \& Wilcots, E. 2011, ApJ, 738, 145

Foley, A., et al., accepted to MNRAS

Geller, M. J., \& Huchra, J. P. 1983, ApJS, 52, 61

Giovanelli, R., Haynes, M. P., Kent, B. R., et al. 2005, AJ, 130, 2598

Haynes, M. P., \& Giovanelli, R. 1984, AJ, 89, 758

Haynes, M. P., Giovanelli, R., Martin, A. M., et al. 2011, AJ, 142, 170

Heitsch, F., \& Putman, M. E. 2009, ApJ, 698, 1485

Hess, K. M., \& Wilcots, E. M. 2013, AJ, 146, 124

Hess, K. M., Jarrett, T. H., Carignan, C., Passmoor, S. S., \& Goedhart, S. 2015, MNRAS, 452, 1617

Hibbard, J. E., Vacca, W. D., \& Yun, M. S. 2000, AJ, 119, 1130

Hibbard, J. E., van Gorkom, J. H., Rupen, M. P., \& Schiminovich, D. 2001, Gas and Galaxy Evolution, 240,

Hickson, P. 1982, ApJ, 255, 382

Hickson, P., Mendes de Oliveira, C., Huchra, J. P., \& Palumbo, G. G. 1992, ApJ, 399, 353

Huang, S., Haynes, M. P., Giovanelli, R., \& Brinchmann, J. 2012, ApJ, 756, 113

Huchtmeier, W. K. 1997, A\&A, 325, 473

Jaffé, Y. L., Poggianti, B. M., Verheijen, M. A. W., Deshev, B. Z., \& van Gorkom, J. H. 2013, MNRAS, 431, 2111

Jaffé, Y. L., Smith, R., Candlish, G. N., et al. 2015, MNRAS, 448, 1715

Johnson, K.E., Hibbard, J.E., Gallagher, S.C., Charlton, J.C., Hornschemeier, A.E., Jarrett, T.H.\& Reines, A.E. 2007, AJ, 134, 1522

Kilborn, V. A., Forbes, D. A., Koribalski, B. S., Brough, S., \& Kern, K. 2006, MNRAS, 371, 739

Koribalski, B., \& Dickey, J. M. 2004, MNRAS, 348, 1255

Leisman, L., Haynes, M. P., Giovanelli, R., et al. 2016, MNRAS, accepted

Makarov, D., \& Karachentsev, I. 2011, MNRAS, 412, 2498

Maloney, P. 1993, ApJ, 414, 41

Martínez, M. A., Del Olmo, A., Coziol, R., \& Perea, J. 2010, AJ, 139,1199

McMullin, J. P., Waters, B., Schiebel, D., Young, W., \& Golap, K. 2007, Astronomical Data Analysis Software and Systems XVI, 376, 127

Nulsen, P.E.J. 1982, MNRAS, 198, 1007

Ogle, P., Boulanger, F., Guillard, P., et al. 2010, ApJ, 724, 1193

Osmond, J. P. F., \& Ponman, T. J. 2004, MNRAS, 350, 1511

Ponman, T. J., Bourner, P. D. J., Ebeling, H., Böhringer, H. 1996, MNRAS, 283, 690

Putman, M. E., Peek, J. E. G., \& Joung, M. R. 2012, ARA\&A, 50,491

Quilis, V., Moore, B. \& Bower 2000, Science, 288, 1617

Rasmussen, J., Ponman, T. J., Verdes-Montenegro, L., Yun, M. S., \& Borthakur, S. 2008, MNRAS, 388, 1245

Robotham, A. S. G., Norberg, P., Driver, S. P., et al. 2011, MNRAS, 416, 2640

Serra, P., Koribalski, B., Kilborn, V., et al. 2015, MNRAS, 452, 2680

Serra, P., Koribalski, B., Duc, P.-A., et al. 2013, MNRAS, 428, 370

Serra, P., Oosterloo, T., Morganti, R., et al. 2012, MNRAS, 422, 1835

Springob, C. M., Haynes, M. P., Giovanelli, R., \& Kent, B. R. 2005, ApJS, 160, 149
Sorgho, A., Hess, K. M., Carignan, C., Oosterloo, T., 2016, MNRAS, accepted.

Stanimirovic, S. 2002, Single-Dish Radio Astronomy: Techniques and Applications, 278, 375

Tzanavaris, P., Hornschemeier, A. E., Gallagher, S. C., et al. 2010, ApJ, 716, 556

van Driel, W., Marcum, P., Gallagher, J. S., III, et al. 2001, A\&A, 378,370

van Gorkom, J. 1991, Atoms, Ions and Molecules: New Results in Spectral Line Astrophysics, 16, 1

van Moorsel, G. A. 1988, A\&A, 202, 59

van Zee, L., Maddalena, R. J., Haynes, M. P., Hogg, D. E., \& Roberts, M. S. 1997, AJ, 113, 1638

Verdes-Montenegro, L., Yun, M.S., Williams, B.A., Huchtmeier, W.K., Del Olmo, A., \& Perea, J. 2001, A\&A, 377, 812

Verdes-Montenegro, L., Yun, M. S., Perea, J., del Olmo, A., \& Ho, P. T. P. 1998, ApJ, 497, 89

Walker, L. M., Johnson, K. E., Gallagher, S. C., et al. 2012, AJ, 143, 69

Walker, L. M., Johnson, K. E., Gallagher, S. C., Hibbard, J. E., Hornschemeier, A. E., Tzanavaris, P., Charlton, J. C., \& Jarrett, T. H. 2010, AJ, 140, 1254

Westmeier, T., Braun, R., \& Koribalski, B. S. 2011, MNRAS, 410, 2217

Williams, B. A., \& Rood, H. J. 1987, ApJS, 63, 265

Williams, B. A., McMahon, P. M., \& van Gorkom, J. H. 1991, AJ, 101, 1957

Yun, M. S., Ho, P. T. P., \& Lo, K. Y. 1994, Nature, 372, 530

This paper has been typeset from a $\mathrm{T}_{\mathrm{E}} \mathrm{X} / \mathrm{LAT} \mathrm{TX}$ file prepared by the author. 